\title{
ARTICLE
}

Epidemiology

\section{Reproductive factors, exogenous hormone use and incidence of melanoma among women in the United States}

\author{
Grayson M. Donley ${ }^{1}$, Wayne T. Liu ${ }^{1}$, Ruth M. Pfeiffer ${ }^{1}$, Emily C. McDonald ${ }^{1}$, Kamau O. Peters ${ }^{1}$, Margaret A. Tucker ${ }^{1}$ and \\ Elizabeth K. Cahoon ${ }^{1}$
}

BACKGROUND: Although the photosensitising effects of oestrogens may increase the impact of ultraviolet radiation (UVR) on melanoma risk, few prospective studies have comprehensively assessed the association between oestrogen-related factors and melanoma.

METHODS: We examined the associations between reproductive factors, exogenous oestrogen use and first primary invasive melanoma among 167503 non-Hispanic white, postmenopausal women in the NIH-AARP Diet and Health Study. Satellite-based ambient UVR estimates were linked to geocoded residential locations of participants at study baseline.

RESULTS: Increased risk of melanoma was associated with early age at menarche ( $\leq 10$ vs $\geq 15$ years: $\mathrm{HR}=1.25,95 \% \mathrm{Cl}: 0.92,1.71$; $P$ for trend $=0.04$ ) and late age at menopause ( $\geq 50$ vs $<45$ years: $\mathrm{HR}=1.34,95 \% \mathrm{Cl}: 1.13,1.59 ; P$ for trend $=0.001)$. The relationship between ambient UVR and melanoma risk was highest among women with age at menarche $\leq 10$ years (HR per UVR quartile increase $=1.29 ; 95 \% \mathrm{Cl}: 1.05,1.58 ;$-interaction $=0.02$ ). Melanoma risk was not associated with parity, age at first birth, use of oral contraceptives or use of menopausal hormone therapy.

CONCLUSIONS: Our findings suggest that increased melanoma risk is associated with early age at menarche and late age at menopause. Effect modification findings support the hypothesis that endogenous oestrogen exposure in childhood increases photocarcinogenicity. Future studies should include information on personal UVR exposure and sun sensitivity.

British Journal of Cancer (2019) 120:754-760; https://doi.org/10.1038/s41416-019-0411-z

\section{BACKGROUND}

Cutaneous melanoma is the fifth most common cancer in the United States with an estimated 87,110 new cases and 9730 deaths expected in 2017. ${ }^{1,2}$ Well-established risk factors for melanoma include ultraviolet radiation (UVR) exposure, ${ }^{3-5}$ pigmentary traits, ${ }^{6,7}$ melanocytic nevi, ${ }^{8,9}$ family history of melanoma (including high- and low-risk susceptibility genes ${ }^{6,10}$ ), inherited genetic conditions ${ }^{11}$ and older age. ${ }^{7,10}$ The age-specific incidence of melanoma is slightly greater among women than among men in the US until the age of 50 years, at which point the rates of melanoma in men begin to increase sharply, while incidence rates in women tend to level off. ${ }^{12}$ The observation of these sex differences in the US and Europe, ${ }^{13-15}$ historical case reports of melanoma diagnosed during pregnancy, ${ }^{16}$ case reports of photosensitivity following oestrogen use including spectrophotometer testing confirming a high UV absorption for the administered oestrogen compound ${ }^{17,18}$ and findings of oestrogen receptors in melanoma lesions ${ }^{19,20}$ have motivated a number of epidemiological studies examining both endogenous oestrogen exposure (e.g. age at menarche, parity) and exogenous oestrogen use (e.g., oral contraceptives [OCs], menopausal hormone therapy [MHT]).

Epidemiological studies evaluating the associations between oestrogen-related factors and melanoma risk have been inconsistent. A 2011 meta-analysis examining a variety of reproductive factors and exogenous hormone use found few pooled associations between melanoma and most oestrogen-related factors. ${ }^{21}$ However, increased melanoma risk was reported for older age at first birth, while decreased risk was found for parity, findings which could potentially be explained by reduced sun exposure among women with children. ${ }^{21}$ Most of the epidemiological studies to date have been either case-control studies ${ }^{21,22}$ or cohort studies in countries with relatively low levels of ambient UVR. $^{21,23,24}$ In addition, few studies have examined whether oestrogen-related factors modify the relationship between UVR exposure and melanoma. ${ }^{23,25}$

Here we assess the associations between reproductive factors, exogenous oestrogen use and subsequent risk of melanoma among female participants of the National Institutes of Health (NIH)-AARP Diet and Health Study. To our knowledge, this is the largest prospective study to evaluate a broad range of reproductive factors and exogenous oestrogen use in relation to melanoma among US women exposed to substantial variation in ambient UVR.

\section{MATERIALS AND METHODS}

Overview

Detailed descriptions of the NIH-AARP Diet and Healthy Study cohort and methods have been previously published. ${ }^{26}$ Briefly, a

${ }^{1}$ Division of Cancer Epidemiology and Genetics, National Cancer Institute, National Institutes of Health, U.S. Department of Health and Human Services, Rockville, MD, USA Correspondence: Grayson M. Donley (grayson.donley@nih.gov)

Received: 8 August 2018 Revised: 5 February 2019 Accepted: 8 February 2019

Published online: 28 February 2019 
self-administered questionnaire was sent to 3.5 million AARP members aged 50-71 years in six states (California, Florida, Pennsylvania, New Jersey, North Carolina, and Louisiana) and two metropolitan areas (Atlanta, Georgia and Detroit, Michigan) between 1995 and 1996. The baseline questionnaire gathered information on demographic characteristics, health-related behaviours and dietary intake. A second, risk factor questionnaire was administered between 1996 and 1997, collecting more detailed information on MHT use. The NIH-AARP study was approved by the Special Studies Institutional Review Board of the National Cancer Institute.

\section{Study population}

Our study population included female, non-Hispanic white, postmenopausal participants of the NIH-AARP Diet and Healthy Study. Of the 566,398 participants who completed the baseline questionnaire, we serially excluded those with questionnaires filled out by proxies $(n=15,760)$, male respondents $(n=325,171)$, participants who died or were diagnosed with any cancer (excluding nonmelanoma skin cancer) before entry $(n=25,428)$, non-white participants $(n=21,867)$, women who were premenopausal at baseline $(n=9569)$, participants whose periods stopped due to radiation or were aged $<60$ years with no listed reason for menopause $(n=867)$, individuals without person-time at risk $(n=11)$ and individuals with missing information on UVR $(n=222)$. The resulting analytic cohort consisted of 167,503 participants. We also examined the associations between melanoma risk and detailed MHT use among a subset of women who also responded to the second questionnaire $(n=108,295)$.

Cohort follow-up and case ascertainment

Participants were followed from their age at the completion of the baseline questionnaire (or the second risk factor questionnaire for analyses of detailed MHT use) until age of the earliest of cancer diagnosis, death, most recent linkage with cancer registries (December 31, 2011) or age when the participant moved out of the registry area. Incident melanoma cases were identified via probabilistic record linkage with state cancer registries and defined using the International Classification of Disease for Oncology, Third edition by anatomic site (C44.0-C44.9) and histology (8720-8780). This classification excludes melanomas described as metastases. Melanoma in situ and invasive melanoma were treated as separate outcomes and women with an incident melanoma in situ were censored in analyses for invasive melanoma. Invasive melanoma will henceforth be referred to simply as melanoma.

\section{Exposure and covariate assessment}

Reproductive factors and exogenous oestrogen use were ascertained from the baseline questionnaire, which additionally collected information on education, marital status, body mass index (BMI), physical activity in the last 12 months, physical activity during adolescence (ages 15-18 years), smoking status, family history of cancer, age at menarche, parity, age at first live birth, menopausal reason, age at menopause, OC use and duration, MHT use and duration and status of surgical ovary removal. The followup risk factor questionnaire ascertained information on receiving a colonoscopy or sigmoidoscopy in the previous 3 years and detailed information on MHT use including oestrogen/progestin vs oestrogen only, dose, and duration.

Ground-level ambient UVR exposure for participants was assigned via linkage of geocoded residential location at study baseline to the Total Ozone Mapping Spectrometer database maintained by the National Aeronautics and Space Administration. $^{27,28}$ This database provides daily estimates of noon-time erythemal UVR exposure on a $1^{\circ}$ latitude by $1.25^{\circ}$ longitude grid. Ground-level erythemal exposure was averaged across all available measured days in the month of July between 1978-1993 and
1996-2005, as levels of ambient UVR have remained relatively stable over time ${ }^{29}$ and surface UVR is strongest during the summer. ${ }^{30}$

\section{Statistical analysis}

To assess the relationship between reproductive factors, exogenous oestrogen use and first primary melanoma, hazard ratios (HRs) and $95 \%$ confidence intervals (Cls) were computed using Cox proportional hazard regression analyses with age as the timescale. Follow-up started at age of entry into the cohort. The following variables were considered potential confounders because they could be significantly associated with both reproductive factors/ exogenous oestrogen use and melanoma incidence but not believed to be on the causal pathway: birth cohort, education, marital status, BMI, smoking history, alcohol consumption, physical activity, coffee consumption, family history of cancer, colonoscopy/sigmoidoscopy in past 3 years, OC/MHT use (for reproductive factors), and ambient UVR. Although no direct measure of skin cancer screening was available in our data, age, education, marital status and colonoscopy/sigmoidoscopy in the past 3 years were examined as correlate of health-care utilisation to account for possible differences in medical surveillance. We also examined whether mutual adjustment for reproductive factors and exogenous hormones changed coefficients. Adjusted models included potential confounders that changed the risk coefficients by at least $15 \%$.

Final models for reproductive factors were adjusted for age, education (less than high school, high school, some college, college, or graduate school), BMI $(>18.5-<25,25-<30$, $30-<60 \mathrm{~kg} / \mathrm{m}^{2}$ ), smoking status (never, former, current), marital status (married, widowed, divorced/separated, never married), family history of cancer (no, yes), colonoscopy/sigmoidoscopy (no, yes), MHT use (no, yes) and ambient UVR quartile (coded continuously 1-4). Final models for exogenous hormones were adjusted for age, education, BMl, smoking status (never, former, current), marriage (married, widowed, divorced/separated, never married), family history of cancer, colonoscopy/sigmoidoscopy, ever use of MHT (for OC models) and ambient UVR quartile. Missing or extreme values for covariates were coded as separate categories and included as indicator variables in the models. To address the hypothesis that oestrogen-related factors are photosensitising, ambient UVR was tested for multiplicative interaction with reproductive factors and exogenous hormone use.

We conducted several additional analyses. We investigated the association between melanoma risk and detailed MHT use among a subset of women who also responded to the second survey. We also examined whether updating information on MHT use to women who responded to the second survey using a timedependent variable for MHT (never, past, current) impacted our findings. To assess the potential impact of medical surveillance/ skin cancer screening, we explored the relationship between reproductive factors, exogenous oestrogen use and risk of melanoma in situ. The proportional hazards assumption was satisfied for all hazard models. Statistical tests were two-sided with a significance level of $a=0.05$. Analyses were conducted using the SAS 9.4 software (SAS Institute, Cary, NC).

\section{RESULTS}

The study population included 167,503 non-Hispanic white, postmenopausal women who were cancer free at baseline. Over a median follow-up time of 15.5 years, $0.6 \%$ of eligible participants had an incident melanoma $(n=1061)$. Women with melanoma were of a similar age at study entry as women without melanoma (Table 1). A greater proportion of women with melanoma reported engaging in physical activity three or more times per week than women without melanoma, both in the past 12 months or during adolescence. 
Table 1. Distribution of selected characteristics of women with and without melanoma in the NIH-AARP Diet and Health Study

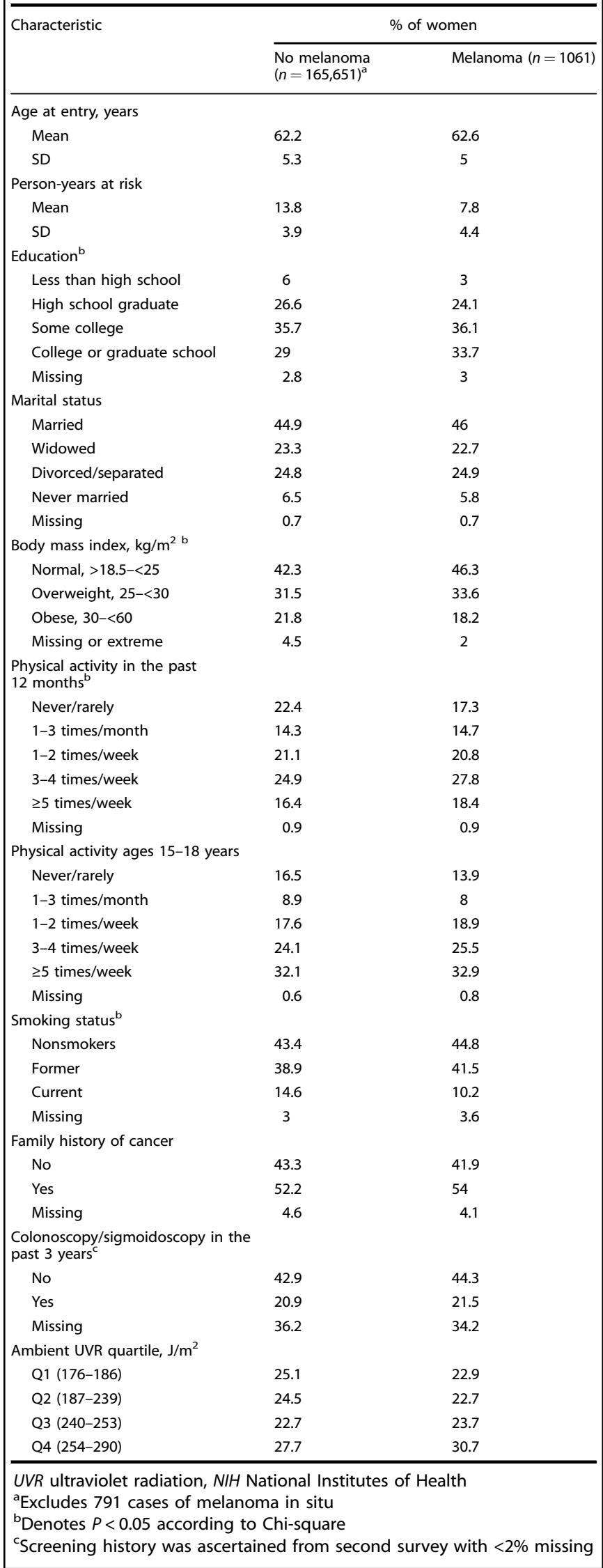

We found an increased risk of melanoma among women who experienced menarche at an early age (age $\leq 10$ vs $\geq 15$ years: $\mathrm{HR}=1.25,95 \% \mathrm{Cl}: 0.92,1.71 ; P$ for trend $=0.04)$, but no associations between age at first live birth or parity (Table 2). Late age at menopause increased melanoma risk (age $\geq 50$ vs $<45$ : $\mathrm{HR}=1.34,95 \% \mathrm{Cl}: 1.13,1.59 ; P$ for trend $=0.001)$. When we stratify women by menopausal reason, we found a positive relationship between both age at natural menopause and melanoma risk (age $\geq 50$ vs $40-44$ years: $\mathrm{HR}=1.30,95 \% \mathrm{Cl}: 0.94,1.79 ; P$ for trend $=0.01$ ) and age at surgical menopause and melanoma risk (age $\geq 50$ vs $40-44$ years: $\mathrm{HR}=1.11,95 \% \mathrm{Cl}$ : $0.85,1.47 ; P$ for trend $=0.005$ ). Use of exogenous oestrogen or duration of use (at baseline) was not associated with melanoma (Table 3). These findings did not change when updating MHT use among women who also responded to the second survey. Analyses using detailed information on formulation of MHT collected on the second survey (i.e. oestrogen/progestin) also did not reveal any associations (Supplementary Table 1).

The association between ambient UVR and melanoma risk was modified by age at menarche ( $P$ for interaction $=0.02)$, with the strongest association observed among women with early age at menarche (Fig. 1).

The relationship between UVR exposure and melanoma incidence was non-significantly higher among ever OC users $(P$ for interaction $=0.09$ ). The effect of UVR on melanoma incidence was not modified by age at menopause or MHT use (Supplementary Table 2).

To assess the sensitivity of our findings to the potential impact of skin cancer screening, we also examined the associations between oestrogen-related factors and risk of melanoma in situ. We found no associations between melanoma in situ and reproductive factors (Supplementary Table 3) or exogenous oestrogen use (Supplementary Table 4).

\section{DISCUSSION}

In this large cohort study, we prospectively evaluated the relationship between reproductive factors, exogenous oestrogen use and melanoma risk in US women residing in areas with substantial variation in ambient UVR. Early age at menarche and late age at menopause were associated with increased risk of melanoma. The association between ambient UVR and melanoma was strongest among women who experienced menarche at an early age. To our knowledge, this is the first study to demonstrate effect modification by age at menarche for the relationship between baseline ambient UVR and melanoma risk.

Melanoma risk was associated with early age at menarche and late age at menopause, supporting the hypothesis that oestrogen exposure is photocarcinogenic. ${ }^{17,31}$ Endogenous oestrogen levels are highest during the ovulation period, which begins at menarche and decreases sharply during the menopausal transition. ${ }^{32}$ Our results are consistent with another cohort study which found that shorter lifetime duration of ovulation was associated with decreased risk of melanoma among women living in France. $^{25}$ However, a meta-analysis consisting primarily of case-control studies found no association between age at menarche, age at menopause and melanoma risk. ${ }^{21}$

We found no relationship between parity or age at first live birth and melanoma risk. These findings are in contrast to a 2011 metaanalysis that reported lower parity and higher age at first birth to be associated with risk of melanoma. ${ }^{21}$ The authors of the metaanalysis proposed that their findings could be due to residual confounding by socioeconomic or lifestyle factors related to individual sun exposure. ${ }^{21}$ A later prospective cohort study in France found a marginally decreased risk of melanoma among women with $>3$ births. ${ }^{25}$ The authors noted that the association between high parity and melanoma risk was somewhat stronger among premenopausal women, who were not included in our 
Table 2. Reproductive factors and risk of melanoma among women in the NIH-AARP Diet and Health Study

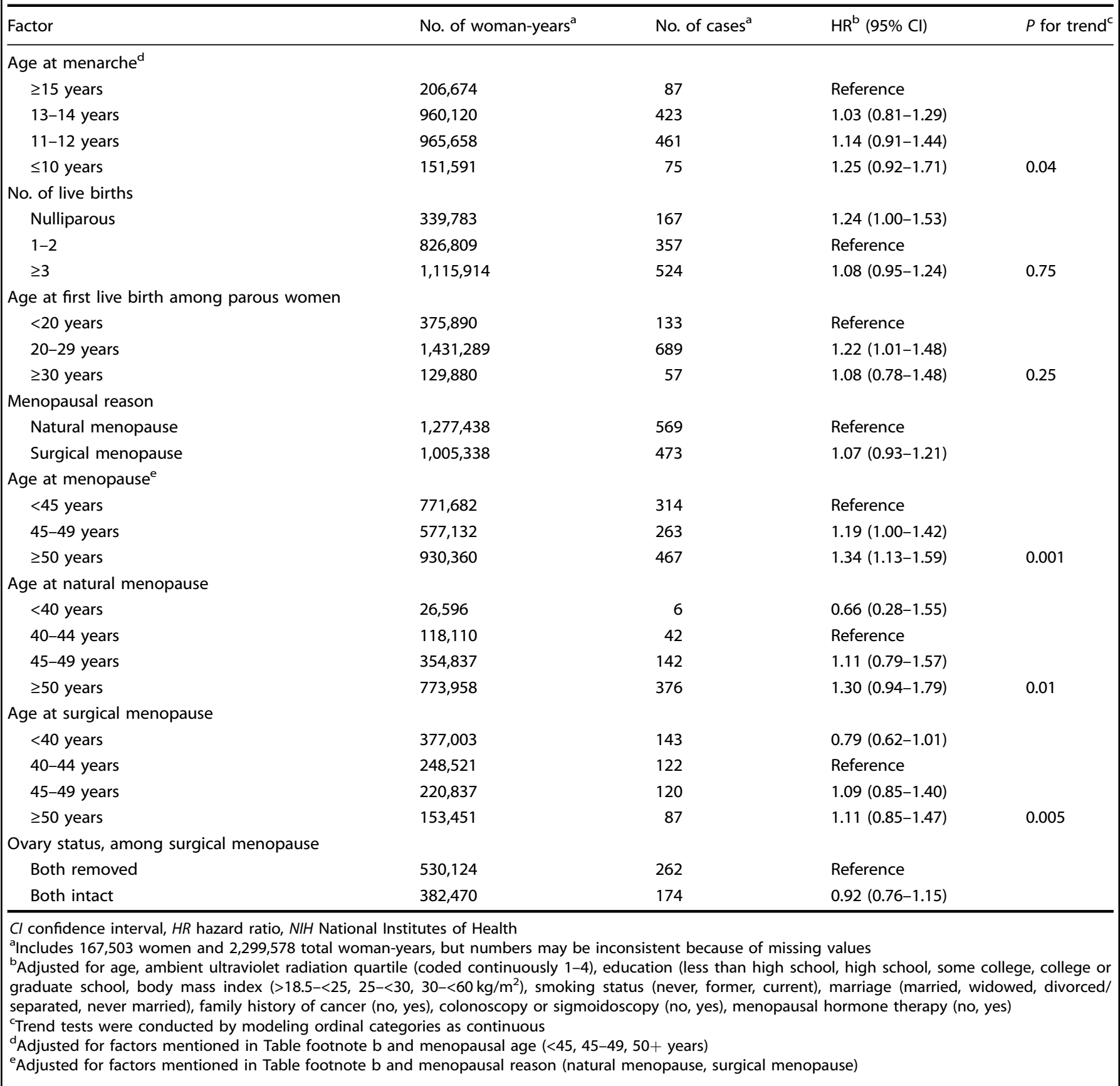

analysis. ${ }^{25}$ A large prospective cohort study in Denmark confirmed associations between parity, younger age at first birth and decreased melanoma risk for both women and men, lending support to the explanation that these associations might reflect confounding by lifestyle or socioeconomic factors. ${ }^{24}$ Our null findings are based on models adjusted for a number of lifestyle and socioeconomic factors (e.g. BMl, physical activity and education) but do not include individual measures of sun exposure or time outdoors. In addition, while pregnancy is associated with increased oestrogen exposure, endogenous oestrogen exposure decreases from breastfeeding. A lack of information on breastfeeding limits our ability to further interpret these null results.

Exogenous oestrogen use was not associated with risk of melanoma in our cohort. This finding agrees with previous research including a meta-analysis of seven case-control studies and three cohort studies that demonstrated no increased melanoma risk associated with $O C$ use. ${ }^{21}$ However, a large case-control study in the Netherlands reported that melanoma was associated with both $\mathrm{OC}$ and $\mathrm{MHT}$ use. ${ }^{22} \mathrm{~A}$ recent large, prospective study of Norwegian women also reported a positive association between oestrogen-only MHT and melanoma risk but no association with oestrogen and progestin MHT and melanoma. ${ }^{23}$ Our findings are consistent with the US Women's Health Initiative clinical trial, which found no association between MHT use and melanoma among white, postmenopausal women aged $50-79$ years (though based on just 95 cases). ${ }^{33}$

We are the first to report effect estimates between current residential UVR and melanoma risk across strata of various hormone-related factors. Notably, melanoma risks associated with 
G.M. Donley et al.

Table 3. Exogenous oestrogen use and risk of melanoma among women in the NIH-AARP Diet and Health Study

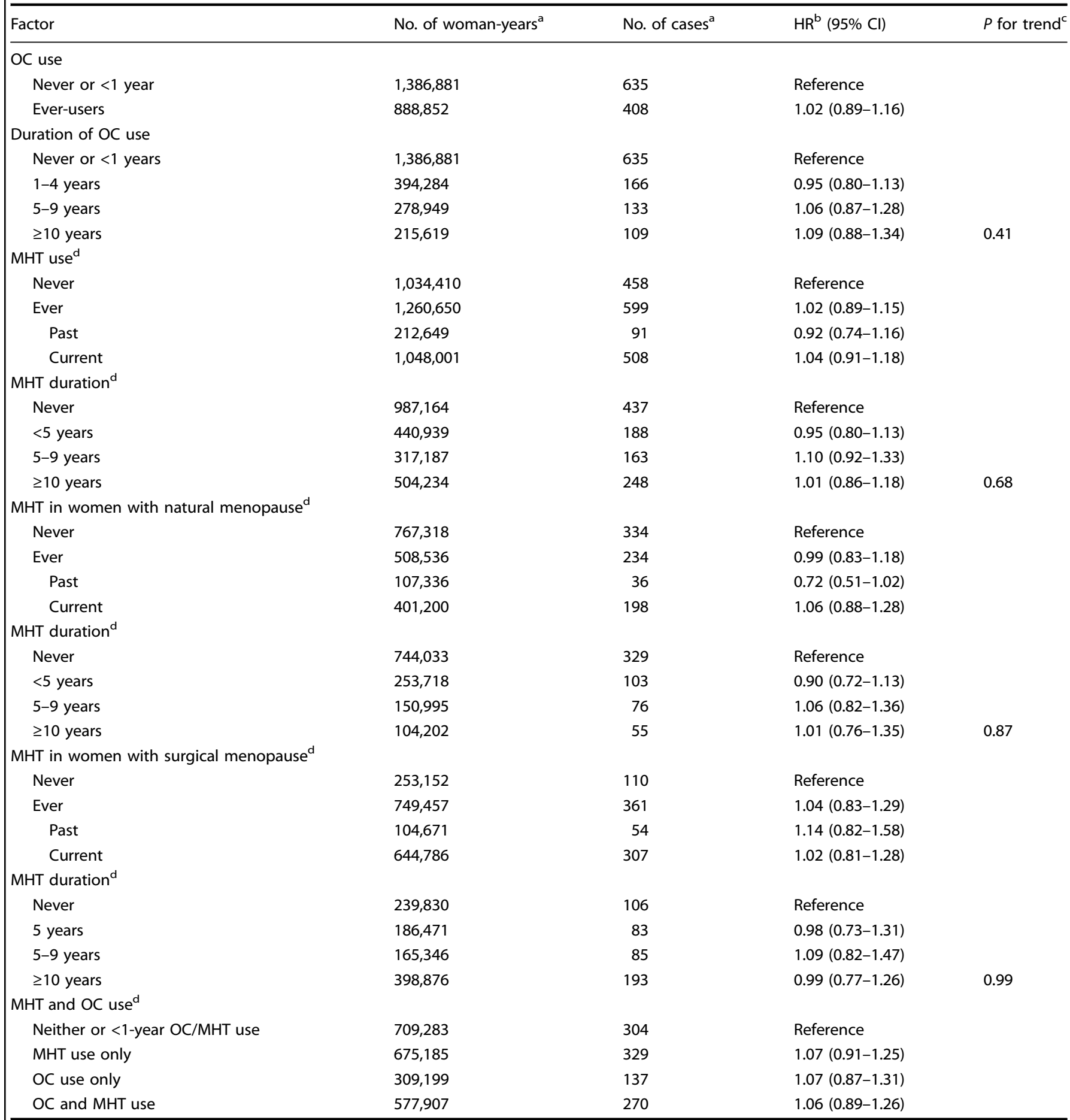

$\mathrm{Cl}$ confidence interval, $\mathrm{HR}$ hazard ratio, OC oral contraceptive, $M H T$ menopausal hormone therapy, NIH National Institutes of Health

ancludes 167,503 women and 2,299,578 total woman-years, but numbers may be inconsistent because of missing values

${ }^{\mathrm{b}}$ Adjusted for age, ambient ultraviolet radiation quartile (coded continuously 1-4), education (less than high school, high school, some college, college or graduate school, body mass index $\left(>18.5-<25,25-<30,30-<60 \mathrm{~kg} / \mathrm{m}^{2}\right)$, smoking status (never, former, current), marriage (married, widowed, divorced/ separated, never married), family history of cancer (no, yes), colonoscopy or sigmoidoscopy (no, yes), menopausal hormone therapy (no, yes)

'Trend tests were conducted by modeling ordinal categories as continuous

${ }^{d}$ Adjusted for factors mentioned in Table footnote b except for menopausal hormone therapy (no, yes)

ambient UVR were highest in women with early age at menarche and among women who used OCs. Melanoma risk is believed to be most strongly associated with early-life sun exposure. ${ }^{7,34-37}$ The findings of effect modification by age at menarche and OC use, but not age at menopause or MHT use, underpin the importance of early-life oestrogen exposure for melanoma risk, especially among women living in locations with high levels of ambient UVR. Unfortunately, our study only had access to ambient UVR at 
a

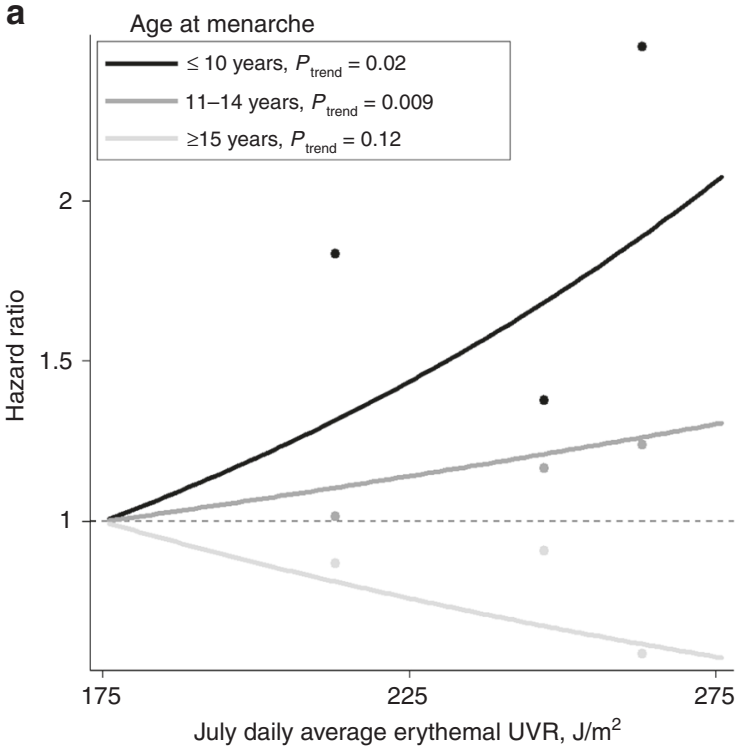

b

Fig. 1 Risk of melanoma among non-Hispanic white, postmenopausal women according to ultraviolet radiation (UVR) exposure and a age at menarche and $\mathbf{b}$ oral contraceptive (OC) use. Solar ambient UVR points represent quartiles of exposure $\left(\mathrm{J} / \mathrm{m}^{2}\right)$. Trend and interaction $P$ values are based on UVR quartile (coded continuously 1-4). $P$ for interactions are 0.02 for age at menarche and 0.09 for OC use. Lines plotted using continuous UVR

baseline and did not have a UVR measure for childhood residence. To our knowledge, only two previous studies examined effect modification between UVR and factors related to endogenous oestrogen and hormone use for melanoma. ${ }^{23,25}$ Kvaskoff et al. found no evidence of interaction between endogenous oestrogens, ambient UVR and melanoma risk in a French cohort. ${ }^{25}$ Botteri et al. also reported that the association between MHT dose and melanoma risk did not vary across UVR levels in Norway. ${ }^{23}$ However, the US women in our large prospective study were exposed to a very broad range of ambient UVR.

This study has several limitations beyond those previously mentioned. We did not have access to a direct measure of skin cancer screening, which may induce a medical surveillance bias in analyses of prescribed hormone use and melanoma risk. Recently, increased screening (e.g. skin biopsy) has been linked to increases in diagnoses of melanoma in situ among the elderly US population. ${ }^{38}$ However, our findings of no association between exogenous hormone use or reproductive factors and melanoma in situ do not suggest a strong influence of medical surveillance/ skin cancer screening bias in this cohort. Since the age of participants at the start of follow-up was relatively high (50-71 years), only late-onset melanoma could be examined. We also lacked information on pigmentation characteristics, including complexion, skin colour, eye colour, number of nevi and markers of genetic susceptibility. These risk factors were not likely strong confounders in the relationship between oestrogen-related factors and melanoma among non-Hispanic white women but cannot be ruled out as potentially important mediators of the association of photocarcinogenesis.

In summary, early age at menarche and late age at menopause were associated with increased risk of melanoma in this large, geographically dispersed cohort. The positive association between ambient UVR and melanoma risk was especially strong among women who experienced menarche at age $\leq 10$ years and women who reported OC use, reinforcing the importance of early-life exposures for cutaneous melanoma. These finding suggest that women with longer cumulative exposure to endogenous oestrogens may benefit from more frequent skin cancer screening, especially those residing in areas with elevated levels of ambient UVR. Future studies should incorporate measures of personal lifetime UVR exposure, detailed sun-susceptibility information, information on breastfeeding and, ideally, longitudinal biological measurements of circulating oestrogens.

\section{ACKNOWLEDGEMENTS}

This research was supported in part by the Intramural Research Program of the National Cancer Institute at the National Institutes of Health. Cancer incidence data were collected by the Georgia Center for Cancer Statistics, Department of Epidemiology, Rollins School of Public Health, Emory University (for the Atlanta metropolitan area); the California Department of Health Services, Cancer Surveillance Section; the Michigan Cancer Surveillance Program, Community Health Administration, State of Michigan (for the Detroit metropolitan area); the Florida Cancer Data System (FCDC) under contract with the Florida Department of Health (FDOH); the Louisiana Tumor Registry, Louisiana State University Medical Center in New Orleans; the New Jersey State Cancer Registry, Cancer Epidemiology Services, New Jersey State Department of Health and Senior Services; the North Carolina Central Cancer Registry; the Division of Health Statistics and Research, Pennsylvania Department of Health, Harrisburg, Pennsylvania; the Arizona Cancer Registry, Division of Public Health Services, Arizona Department of Health Services; and the Texas Cancer Registry, Cancer Epidemiology and Surveillance Branch, Texas Department of State Health Services. The Pennsylvania Department of Health specifically disclaims responsibility for any analyses, interpretations or conclusions. The views expressed herein are solely those of the authors and do not necessarily reflect those of the FCDC or FDOH.

\section{AUTHOR CONTRIBUTIONS}

All authors of this manuscript have participated in the planning, execution, or analysis of this study and approved the final version. E.K.C. and W.T.L. designed the study, E.K.C., G.M.D., and W.T.L. assembled the data, analysed the data, and drafted the manuscript. R.M.P., E.C.M., K.O.P., and M.A.T. interpreted the analysis and critically revised the paper.

\section{ADDITIONAL INFORMATION}

Supplementary information is available for this paper at https://doi.org/10.1038/ s41416-019-0411-z.

Competing interests: The authors declare no competing interests.

Ethical approval and consent to participate: The NIH-AARP study was approved by the Special Studies Institutional Review Board of the National Cancer Institute. 
Data availability: Materials and data are available from the corresponding author upon reasonable request.

Publisher's note: Springer Nature remains neutral with regard to jurisdictional claims in published maps and institutional affiliations.

\section{REFERENCES}

1. American Cancer Society. Cancer Facts \& Figures 2017 (American Cancer Society, Atlanta, GA, 2017).

2. Howlader, N. et al. (eds). SEER Cancer Statistics Review, 1975-2014 (National Cancer Institute, Bethesda, MD, 2017). http://seer.cancer.gov/csr/1975_2014/.

3. Whiteman, D. C. et al. Anatomic site, sun exposure, and risk of cutaneous melanoma. J. Clin. Oncol. 24, 3172-3177 (2006).

4. Armstrong, B. K. \& Kricker, A. The epidemiology of UV induced skin cancer. J. Photochem. Photobiol. B 63, 8-18 (2001).

5. Gandini, S. et al. Meta-analysis of risk factors for cutaneous melanoma: II. Sun exposure. Eur. J. Cancer 41, 45-60 (2005). .

6. Gandini, S. et al. Meta-analysis of risk factors for cutaneous melanoma: III. Family history, actinic damage and phenotypic factors. Eur. J. Cancer 41, 2040-2059 (2005).

7. Veierod, M. B., Adami, H. O., Lund, E., Armstrong, B. K. \& Weiderpass, E. Sun and solarium exposure and melanoma risk: effects of age, pigmentary characteristics, and nevi. Cancer Epidemiol. Biomark. Prev. 19, 111-120 (2010).

8. Holly, E. A., Kelly, J. W., Shpall, S. N. \& Chiu, S. H. Number of melanocytic nevi as a major risk factor for malignant melanoma. J. Am. Acad. Dermatol. 17, 459-468 (1987).

9. Gandini, S. et al. Meta-analysis of risk factors for cutaneous melanoma: I. Common and atypical naevi. Eur. J. Cancer 41, 28-44 (2005).

10. Begg, C. B. et al. Familial aggregation of melanoma risks in a large populationbased sample of melanoma cases. Cancer Causes Control 15, 957-965 (2004).

11. Read, J., Wadt, K. A. \& Hayward, N. K. Melanoma genetics. J. Med. Genet. 53, 1-14 (2016).

12. Watson, M. et al. Melanoma surveillance in the United States: overview of methods. J. Am. Acad. Dermatol. 65(5 Suppl 1), S6-S16 (2011).

13. Roh, M. R., Eliades, P., Gupta, S., Grant-Kels, J. M. \& Tsao, H. Cutaneous melanoma in women. Int. J. womens Dermatol. 3(1 Suppl), S11-S15 (2017).

14. de Vries, E. et al. Superior survival of females among 10,538 Dutch melanoma patients is independent of Breslow thickness, histologic type and tumor site. Ann. Oncol. 19, 583-589 (2008).

15. Gamba, C. S., Clarke, C. A., Keegan, T. H., Tao, L. \& Swetter, S. M. Melanoma survival disadvantage in young, non-Hispanic white males compared with females. JAMA Dermatol. 149, 912-920 (2013).

16. Andersson, T. M., Johansson, A. L., Fredriksson, I. \& Lambe, M. Cancer during pregnancy and the postpartum period: a population-based study. Cancer 121, 2072-2077 (2015).

17. Erickson, L. R. \& Peterka, E. S. Sunlight sensitivity from oral contraceptives. JAMA 203, 980-981 (1968)

18. Richarz, N. A. et al. Phototoxic reaction to a combined oral contraceptive (levonorgestrel/ethinylestradiol). Photochem. Photobiol. Sci. 16, 1381-1383 (2017).

19. Chaudhuri, P. K., Walker, M. J., Briele, H. A., Beattie, C. W. \& Gupta, T. K. Incidence of estrogen receptor in benign nevi and human malignant melanoma. JAMA 244, 791-793 (1980).

20. Ellis, D. L., Wheeland, R. G. \& Solomon, H. Estrogen and progesterone receptors in primary cutaneous melanoma. J. Dermatol. Surg. Oncol. 11, 54-59 (1985).

21. Gandini, S. et al. Hormonal and reproductive factors in relation to melanoma in women: current review and meta-analysis. Eur. J. Cancer 47, 2607-2617 (2011).

22. Koomen, E. R. et al. Estrogens, oral contraceptives and hormonal replacement therapy increase the incidence of cutaneous melanoma: a population-based case-control study. Ann. Oncol. 20, 358-364 (2009).
23. Botteri, E. et al. Menopausal hormone therapy and risk of melanoma: do estrogens and progestins have a different role? Int. J. Cancer 141, 1763-1770 (2017).

24. Kaae, J., Andersen, A., Boyd, H. A., Wohlfahrt, J. \& Melbye, M. Reproductive history and cutaneous malignant melanoma: a comparison between women and men. Am. J. Epidemiol. 165, 1265-1270 (2007).

25. Kvaskoff, M., Bijon, A., Mesrine, S., Boutron-Ruault, M. C. \& Clavel-Chapelon, F. Cutaneous melanoma and endogenous hormonal factors: a large French prospective study. Am. J. Epidemiol. 173, 1192-1202 (2011).

26. Schatzkin, A. et al. Design and serendipity in establishing a large cohort with wide dietary intake distributions: the National Institutes of Health-American Association of Retired Persons Diet and Health Study. Am. J. Epidemiol. 154, 1119-1125 (2001).

27. Lin, S. W. et al. Prospective study of ultraviolet radiation exposure and risk of cancer in the United States. Int. J. Cancer 131, E1015-E1023 (2012).

28. National Aeronautics and Space Administration. Total Ozone Mapping Spectrometer Data Product: Erythemal UV Exposure (Goddard Space Flight Center, Greenbelt, MD, 2004).

29. Lean, J. L. et al. Detection and parameterization of variations in solar mid- and nearultraviolet radiation (200-400 nm). J. Geophys. Res. 102, 29939-29956 (1997).

30. Ziemke, J. R., Chandra, S., Herman, J. \& Varotsos, C. Erythemally weighted UV trends over northern latitudes derived from Nimbus 7 TOMS measurements. $J$. Geophys. Res. 105, 7373-7382 (2000).

31. Stern, R. S. Photocarcinogenicity of drugs. Toxicol. Lett. 102-103, 389-392 (1998).

32. McKinlay, S. M., Brambilla, D. J. \& Posner, J. G. The normal menopause transition. Maturitas 14, 103-115 (1992).

33. Tang, J. Y. et al. Menopausal hormone therapy and risks of melanoma and nonmelanoma skin cancers: women's health initiative randomized trials. J. Natl. Cancer Inst. 103, 1469-1475 (2011).

34. Wu, S., Han, J., Laden, F. \& Qureshi, A. A. Long-term ultraviolet flux, other potential risk factors, and skin cancer risk: a cohort study. Cancer Epidemiol. Biomark. Prev. 23, 1080-1089 (2014).

35. Weinstock, M. A. et al. Nonfamilial cutaneous melanoma incidence in women associated with sun exposure before 20 years of age. Pediatrics $\mathbf{8 4}, 199-204$ (1989).

36. Whiteman, D. C., Whiteman, C. A. \& Green, A. C. Childhood sun exposure as a risk factor for melanoma: a systematic review of epidemiologic studies. Cancer Causes Control 12, 69-82 (2001).

37. Green, A. C., Wallingford, S. C. \& McBride, P. Childhood exposure to ultraviolet radiation and harmful skin effects: epidemiological evidence. Prog. Biophys. Mol. Biol. 107, 349-355 (2011).

38. Weinstock, M. A. et al. Skin biopsy utilization and melanoma incidence among Medicare beneficiaries. Br. J. Dermatol. 176, 949-954 (2017).

(i) Open Access This article is licensed under a Creative Commons Attribution 4.0 International License, which permits use, sharing, adaptation, distribution and reproduction in any medium or format, as long as you give appropriate credit to the original author(s) and the source, provide a link to the Creative Commons license, and indicate if changes were made. The images or other third party material in this article are included in the article's Creative Commons license, unless indicated otherwise in a credit line to the material. If material is not included in the article's Creative Commons license and your intended use is not permitted by statutory regulation or exceeds the permitted use, you will need to obtain permission directly from the copyright holder. To view a copy of this license, visit http://creativecommons. org/licenses/by/4.0/.

(c) The Author(s) 2019 